Z Herz- Thorax- Gefäßchir 2012 · 26:5-6 DOI 10.1007/s00398-011-0897-z

Online publiziert: 18. Januar 2012

(c) Springer-Verlag 2012
F.-W. Mohr • C.D. Etz

Klinik für Herzchirurgie, Herzzentrum der Universität Leipzig

\section{Sichere Therapie der akuten Typ-A-Dissektion}

\author{
Kanülierungsoptionen, antegrade selektive \\ zerebrale Perfusion und Risiken des distalen \\ Kreislaufstillstands in milder Hypothermie
}

Liebe Leserin, lieber Leser,

die schnelle und sichere Therapie der akuten Typ-A-Dissektion mit Bogenbeteiligung gehört nach wie vor zu den technisch anspruchsvollen Eingriffen der modernen kardiovaskulären Chirurgie. Hier wird der technisch einwandfrei durchgeführte Aorta-ascendens-/Teilbogenersatz aufgrund der komplexen Beteiligung von Aortenklappe sowie Koronarund Kopfarterien anders als beispielsweise im Aorta-descendens-Bereich auch in absehbarer Zukunft den endovaskulären Verfahren überlegen bleiben. Eine konsequent komplette Sanierung reduziert die Notwendigkeit von Zweiteingriffen oder macht diese leichter.

Nachdem Hans G. Borst im Jahr 1964 den ersten Patienten erfolgreich im Kreislaufstillstand operiert, und Randall B. Griepp 1975 von der ersten Eingriffsserie in Bogennähe bei einer Körperkerntemperatur von unter $15^{\circ} \mathrm{C}$ berichtet hatten, trat das Konzept der Verlängerung der Ischämietoleranz der Organsysteme insbesondere des zentralen Nervensystems durch systemische Kühlung und tiefe Hypothermie einen Siegeszug durch die Aortenchirurgie an; die Grundvoraussetzung für die heute recht erfolgreiche Therapie akuter Typ-A-Dissektionen war geschaffen!

Mit der rasanten Entwicklung in der Aortenbogenchirurgie, welche mit der Einführung des tiefen hypothermen Kreislaufstillstands erst begonnen hatte, folgte Ende der 80er Jahre, erstmals in
Europa, die erfolgreiche klinische Wiedereinführung der selektiven zerebralen Perfusion („selective cerebral perfusion“, SCP) zur intraoperativen Gehirnprotektion zunächst in Kombination mit der tiefen Hypothermie, maßgeblich durch Jean Bachet im Jahr 1991 und später durch Teruhisa Kazui in Japan.

Moderne Kanülierungsstrategien mussten also insbesondere diesem neuen Perfusionskonzept Rechnung tragen. So verlor zeitgleich die bis dato als Goldstandard betrachtete, alleinige femorale Kanülierung ungeachtet ihrer schnellen und sicheren Anwendbarkeit an Bedeutung. Die antegrade SCP, die beispielsweise über eine rechte axilläre Perfusion mit oder ohne zusätzliche, endovaskuläre Direktkanülierung mit ballonokkludierbaren Perfusionskathetern der linken A. carotis oder etwa mittels Prothesenbypass sehr effektiv erreicht werden konnte, begann sich zu etablieren.

\section{》) Die tiefe Hypothermie trat zunächst einen Siegeszug durch die Aortenchirurgie an}

Mit einem umfangreichen und detaillierten Überblick über arterielle Kanülierungsoptionen für die akute Typ-A-Dissektion aus Heidelberg startet die vor Ihnen liegende Ausgabe des Journals. Experten der proximalen Aortenchirurgie in Deutschland liefern daneben aus ihrem individuellen Blickwinkel wertvolle Kom- mentare zu speziellen und situativ anwendbaren, modernen Kanülierungsstrategien wie etwa den Zugang über die A. carotis oder die direkte Kanülierung des wahren Lumens in Seldinger-Technik.

Motiviert durch die hervorragenden klinischen Ergebnisse, die mit sicherer Kanülierungstechnik und optimaler Neuroprotektion durch nahezu ununterbrochene Kopfperfusion erzielbar waren, und nicht zuletzt auch durch die unerwünschten Begleiterscheinungen der tiefen Hypothermie (Blutungsneigung, prolongierte Aufwärm- und Operationszeiten und damit einhergehend auch ökonomische Implikationen), prägte die sukzessive Anhebung der Zieltemperatur während dieser komplexen Eingriffe die Entwicklung der bogennahen Aortenchirurgie in der jüngsten Vergangenheit. Ein Trend, ausgehend von kalter antegrader SCP während tiefer Hypothermie, über moderate $\left(28-32^{\circ} \mathrm{C}\right)$ bis hin zur milden $\left(\geq 32^{\circ} \mathrm{C}\right)$ Hypothermie, mit dem Ziel, assoziierte Komplikationen der tiefen Hypothermie zu eliminieren und die zerebrale Autoregulation zu optimieren, setzte ein.

Inmitten der Euphorie angesichts der vielgestaltigen Vorteile dieser neuen Perfusionsstrategie geriet jedoch eines zunehmend aus dem Fokus: das unumgängliche Risiko der prolongierten viszeralen und spinalen Ischämie während des distalen Kreislaufstillstands in nun nur noch moderater bis milder Hypothermie, mit dem verheerenden Effekt der irreversiblen Paraplegie; einer Komplikation, die in 
tiefer Hypothermie bis dato sehr selten ein Problem darstellte.

Nachdem Justus Strauch bereits im Jahr 2003 in Randall Griepps Labor in New York experimentell nachgewiesen hatte, dass die Ischämietoleranz des Rückenmarks von 20 Minuten bei Normothermie auf 50 Minuten bei milder Hypothermie $\left(32^{\circ} \mathrm{C}\right)$ erhöht werden kann, berichteten Kamiya und Mitarbeiter, damals in der Hannoveraner Gruppe tätig, im Jahr 2007 erstmals klinisch über die reale Gefahr neurologischer Komplikationen: in einer Subgruppenanalyse von Patienten mit distalem Körperkreislaufstillstand von mehr als 60 Minuten bei einer Körperkerntemperatur von $28^{\circ} \mathrm{C}$ dokumentierten sie eine Mortalität von $27 \%$ und eine Paraplegierate von 18\% - in tiefer Hypothermie mit SCP für mehr als 60 Minuten lag die Paraplegierate bei $0 \%$.

\section{》) Ist die duale Kanülierung (zentral und peripher) \\ Perfusionsstrategie der Zukunft?}

Christian D. Etz und Mitarbeiter lieferten nur ein Jahr später anlässlich der Jahrestagung der EACTS in Genf erste experimentelle Daten und wiesen nach, dass der distale Kreislaufstillstand während antegrader SCP bei $28^{\circ} \mathrm{C}$ zum kompletten Erliegen der spinalen Perfusion distal der Segmente T8/ T9, mit deletären Folgen für das Rückenmark und die Viszeralorgane, führt und nach 90 Minuten bereits irreversible Ischämieschäden auftreten, während die Paraplegierate nach 120 Minuten bereits 100\% erreicht. Unter Aortenchirurgen schärften diese Ergebnisse die kollektive Wahrnehmung der Problematik des distalen Kreislaufstillstands während milder Hypothermie. Auch diese Thematik wird in dieser Ausgabe der Zeitschrift für Herz-, Thoraxund Gefäßshirurgie detailliert aufgegriffen.

Wollen oder müssen wir diesen Weg weiter gehen, etwa bei dem Patienten mit akut rupturiertem Aorta-ascendens-/Bogenaneurysma oder der akuten dekompensierten Typ-A-Dissektion mit Wandruptur im Bereich des Bogens? Ist die duale zentrale/axillare und zusätzliche periphere/femorale Kanülierung eine mögliche oder sogar notwendige Perfusionsstrategie in der Zukunft?
Insbesondere scheint mir in den Fällen mit komplexem Bogenersatz mit Kompromittierung der Interkostalarterien durch gleichzeitige Stentgraftplatzierung (z.B. „frozen elephant trunk“) die frühzeitige Perfusion der unteren Körperhälfte ratsam; dabei ist die bevorzugte Kanülierungstechnik wichtiger Bestandteil der eigenen Operationsstrategie und bedarf unbedingt der Abstimmung mit Kardiotechnik und Anästhesie.

Das aktuelle Heft unseres Weiterbildungsorgans fasst Vor- und Nachteile verschiedener alternativer Kanülierungsstrategien zusammen. Wir freuen uns über diese rege Diskussion, insbesondere auch hinsichtlich möglicher Risiken und Gefahren des unkritischen Temperaturmanagements während distalen Kreislaufstillstands im Interesse einer optimalen Therapie der akuten Typ-A-Dissektion, deren weltweit führende chirurgische Therapie in Deutschland insbesondere im Hinblick auf ein optimales neurologisches Langzeitergebnis unser Ziel sein sollte!

Ihr

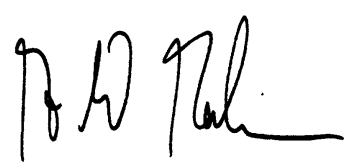

Professor Dr. med. F.-W. Mohr

\section{Korrespondenzadresse}

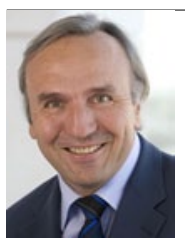

Prof. Dr. FriedrichWilhelm Mohr Klinik für Herzchirurgie, Herzzentrum der Universität Leipzig Strümpelstraße 39 04289 Leipzig mohrf@medizin.uni-leipzig.de

\section{Bei langer Krankheit droht Ärzten Versicherungslücke}

Viele Ärzte, die eine Krankentagegeld- und eine Berufsunfähigkeitsversicherung abgeschlossen haben, gehen davon aus, dass sie bei einer schweren Erkrankung zumindest finanziell auf der sicheren Seite sind. Das kann sich aber als Irrtum herausstellen. In den meisten Fällen ist das Krankentagegeld höher als die Berufsunfähigkeitsdeckung. Das verleitet dazu, den Übergang in die Berufsunfähigkeitsversicherung hinauszuzögern. Die beiden Deckungen greifen jedoch nicht immer nahtlos ineinander. Das Problem: Krankentagegeldversicherungen dienen als Schutz bei einer vorübergehenden Arbeitsunfähigkeit. Die Unternehmen stellen die Leistungen ein, wenn klar ist, dass der Kunde dauerhaft nicht mehr in seinem Beruf arbeiten kann. Meldet sich der Versicherte erst dann beim Berufsunfähigkeitsversicherer oder beim Versorgungswerk, können einige Monate vergehen, bevor die erste Rentenzahlung auf dem Konto eingeht.

Ärzte, die schon länger schwer krank sind sollten daher vorsorglich Leistungen aus der Berufsunfähigkeitsversicherung beantragen. Wenn sich die gesundheitliche Situation bessert, kann man den Antrag problemlos zurückziehen.

Bei den berufsständischen Versorgungswerken ist die Einstufung „berufsunfähig" nicht immer einfach. Denn die Definition, wann ein Arzt seinen Beruf noch ausüben kann, ist weit gefasst. Ärzte, die zusätzlich eine private Berufsunfähigkeitsversicherung abgeschlossen haben, sollten abklären, ob der Berufsunfähigkeitsschutz auf die konkrete zuletzt ausgeübte Tätigkeit abzielt. Es macht einen Unterschied, ob jemand nicht mehr als Arzt tätig sein kann oder nicht mehr in seiner Spezialisierung. Auch sollten sich Ärzte danach erkundigen, ob sie eine Gruppenversicherung für die Berufsunfähigkeit abschließen können. Dort sind die Bedingungen zum Teil besser und auf die spezifischen Bedürfnisse der Klientel abgestimmt.

Quelle: Ärztezeitung, www.aerztezeitung.de 